\title{
Determination of Intrarenal Resistance Index (RI) in Patients with Multiple Myeloma
}

\author{
U. Schiemann ${ }^{1,3}$, H. C. Kaiser ${ }^{1}$, M. Götzberger ${ }^{1}$, R. Schmidmaier ${ }^{2}$, F. Bantle ${ }^{3}$, C. Straka ${ }^{2}$ \\ ${ }^{1}$ Department of Gastroenterology and ${ }^{2}$ Department of Hematology, \\ Medizinische Klinik Innenstadt, Klinikum der Universität München, Germany; \\ ${ }^{3}$ Klinik für Allgemeine Innere Medizin, Inselspital, Bern, Switzerland
}

\begin{abstract}
Background: Renal impairment is a common complication of multiple myeloma occuring in up to $50 \%$ of patients at some stage in their disease. Due to occurrence of cast nephropathies we hypothesized circulatory dysregulation (vasoconstriction) in the kidneys with measurable elevation of the resistance index among these patients which would have a diagnostic impact.

Subjects and methods: 36 patients with treated multiple myeloma (21 females, 15 males, mean age $61.6 \pm 8.5$ years) were prospectively examined by conventional abdominal ultrasound with focussed investigation of the kidneys. First, length of the organs, parenchymal width and characterization of parenchymal echogenicity were determined. Then, intrarenal RI values were measured in segmental and arcuate arteries, respectively, in both kidneys. Additionally, serum creatinine, BUN and GFR of each patient were evaluated. RI values were compared to values of 78 healthy control subjects.

Results: Mean renal RI was $0.68 \pm 0.07$ which was slightly higher than in controls with $0.62 \pm 0.05$, but without statistical significance. Due to the laboratory analyses patients were subdivided in those with normal ( group 1, $\mathrm{n}=21$ ) and those with impaired (group 2, $\mathrm{n}$ $=15$ ) renal function. In both groups kidney size and parenchymal width were normal. Significant more group 2 patients $(60 \%)$ revealed hyperechogenic parenchyma than group 1 patients $(24 \%)(\mathrm{p}<0.01)$. Mean renal RI indices were $0.67 \pm 0.06$ (right) and $0.69 \pm$ 0.06 (left) in group 1 patients and $0.71 \pm 0.08$ (right) and $0.71 \pm 0.07$ (left) in group 2 patients and showed no significant difference $(\mathrm{p}=0.06$ and 0.15$)$.

Conclusion: Renal RI values are not significantly elevated in patients with multiple myeloma even in those with renal impairment so that no hints to a relevant vasoconstriction could be evaluated. RI seems not to be a relevant parameter for the diagnosis of cast nephropathy of multiple myeloma patients. Routinely performed ultrasound examination should be more focussed on the qualification of parenchymal echogenicity.
\end{abstract}

Key words: Resistance Index, multiple myeloma, renal failure, ultrasound
Abbreviations: RI: resistance index; Arc: arcuate arteries; Seg: segmental arteries; BUN: blood urea nitrogen; GFR: glomerular filtration rate

\section{INTRODUCTION}

Renal impairment is a common complication of multiple myeloma occuring in up to $50 \%$ of patients at some stage in their disease $[1,2]$. Exceeded production of monoclonal light chains by multiple myeloma cells and their excretion in the urine as proteinuria (Bence Jones proteins) is the main pathogenetic factor. Later on, tubular atrophy and interstitial fibrosis develops with unfavourable prognosis and requirement of dialysis in $2-3 \%$ of the patients [3-8]. The precipitation of light chains, which form casts in the distal tubules, results in renal obstruction [9] and is the most common cause of renal impairment in multiple myeloma. Due to these parenchymal changes we hypothesized circulatory dysregulation in the kidneys with potential elevation of the resistance index (RI) among multiple myeloma patients. Rivolta and coworkers showed that renal RI correlates with the glomerular filtration rate of the kidneys in patients with liver cirrhosis [10].

Measurement of the intrarenal resistance index has been widely established in the evaluation of renovascular changes. The dopplersonographic RI measurement is a wide-spread method to get indirect information about the vessel resistance and the perfusion distal of the measuring point.

The aim of our study was to perform renal RI measurements in patients with multiple myeloma and to evaluate if these values show a correlation to renal function. Elevated renal RI values as hint to vasoconstrictive effects in the kidneys of these patients would have a diagnostic impact.

\section{SubJeCtS AND METHOdS}

We prospectively examined 36 patients (21 females, 15 males, mean age $61.6 \pm 8.5$ years) of our hematological department with multiple myeloma who were sent to our gastroenterological department for a routine abdominal ultrasound investigation.

After fasting for at least four hours, all subjects were examined by B-mode ultrasound and colour- 


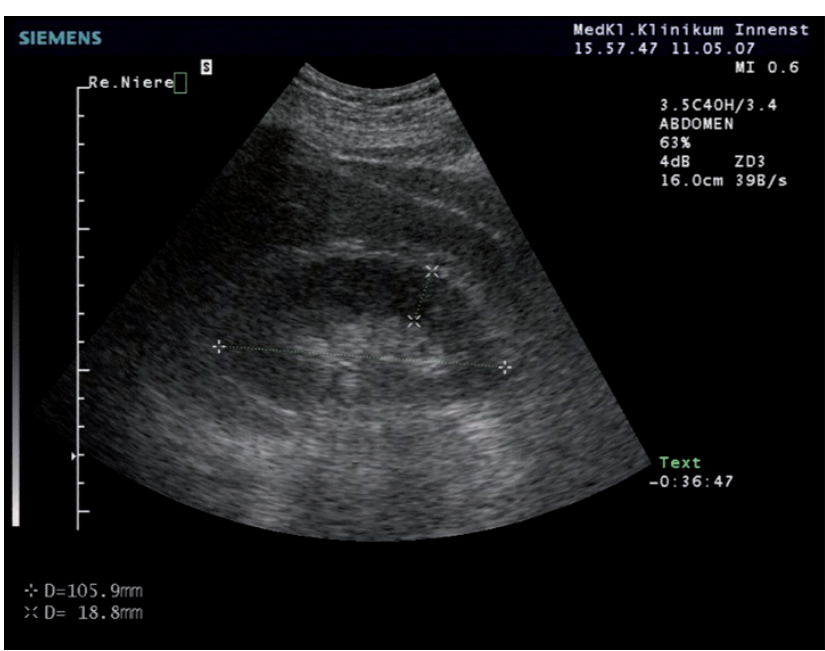

Fig 1. Sonographical measurement of the length $(10.6 \mathrm{~cm})$ and the parenchymal width $(1.9 \mathrm{~cm})$ of the right kidney in a patient with multiple myeloma.

Doppler ultrasound with a $3.5 \mathrm{MHz}$ broadband convex scanner (Siemens SONOLINE Elegra, Erlangen, Germany). Renal length and parenchymal width were determined for both sides (Fig.1).

Colour-flow-doppler signals were taken from segmental arteries near hilum (seg) and arcuate arteries of the cortex (arc) in the right and left kidney, respectively. The examination was done from experienced investigators (US, HK, MG). Intrarenal RI was determined from analysis of the spectral waveform as follows: the peak systolic velocity minus the lowest diastolic velocity divided by the peak systolic velocity (RI = [VmaxSystole-VminDiastole] / VmaxSystole). The height of the pulsed Doppler waveform was maximized to facilitate measurement. The mean RI was calculated as an average of three measurements. Each measurement was done on condition that at least three waveforms followed each other (Fig. 2).

Additionally, serum creatinine, blood urea nitrogen (BUN) and GFR of each patient were determined. Glomerular filtration rate (GFR) was estimated by calculated creatinine clearance.

Informed consent of the patients was given, respectively.

RI values were compared with values of 78 healthy control subjects.

Statistical evaluation was performed by student's ttest. P-values $<0.05$ were considered as statistical significant.

\section{RESULTS}

Mean renal RI was $0.68 \pm 0.07$ which was somewhat higher than in controls with $0.62 \pm 0.05$, but without statistical significance.

Due to the laboratory analyses we subdivided the patients in those with normal serum creatinine, BUN and GFR ( $n=21$, group 1 ) and those with elevated serum creatinine/BUN and decreased GFR ( $\mathrm{n}=15$, group 2).

Mean age of both groups was similar without significant difference (group 1: $60.2 \pm 9.6$ years, group

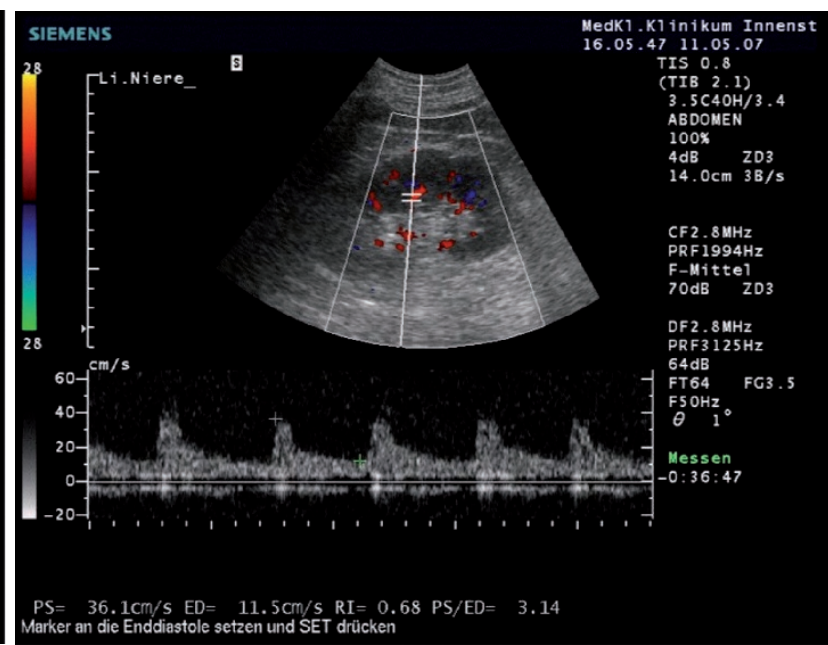

Fig. 2. Measurement of intrarenal resistance index (RI) on a segmental arteria. At least three waveforms following each other were documented. RI is determined with a value of 0.68 .

2:63.4 \pm 6.1. years). All relevant clinical and laboratory data are given in Table 1.

Table 1. Clinical and laboratory data of 36 multiple myeloma patients with normal (group 1) and impaired (group 2) renal function.

\begin{tabular}{lcc}
\hline & Group 1 & Group 2 \\
\hline Number of patients (n) & 21 & 15 \\
Mean age (years) & $60.2 \pm 9.6$ & $63.4 \pm 6.1$ \\
Serum creatinine (mg/dl) & $0.85 \pm 0.2$ & $3.1 \pm 1.1$ \\
BUN (mg/dl) & $13.5 \pm 3.4$ & $41 \pm 23$ \\
GFR (ml/min) & $101 \pm 25$ & $35 \pm 21$ \\
\hline
\end{tabular}

In both groups similar values were measured for kidney length (group 1: right 10.3/left $10.9 \mathrm{~cm}$; group 2: right $10.1 /$ left $10.5 \mathrm{~cm}$ ) and parenchyma width (group 1: right $1.7 /$ left $1.6 \mathrm{~cm}$; group 2: right 1.5/left $1.6 \mathrm{~cm}$ ) ('Table 2).

Only four group 1 patients $(24 \%)$, but nine of group 2 patients $(60 \%)$ revealed hyperechogenicity of renal parenchyma as hint to an interstitial nephropathy. This difference was statistical significant $(\mathrm{p}<0.01)$.

Mean renal RI indices were $0.67 \pm 0.06$ (right) and $0.69 \pm 0.06$ (left) in group 1 patients and $0.71 \pm 0.08$ (right) and $0.71 \pm 0.07$ (left) in group 2 patients. The slight differences were without statistical difference (right $\mathrm{p}=0.06$, left $\mathrm{p}=0.15)($ Table 2$)$.

\section{Discussion}

Measurement of the intrarenal resistance index (RI) has been widely applied in the evaluation of renovascular changes. It is a suitable method for the determination of vasoconstrictive effects in the kidney. Most investigators define a value lower than 0.7 as normal in the adult population. Important to know that the height of RI depends on interobserver-variabilities 
Table 2. Size of the kidneys, parenchymal width and resistance indices (RI) of multiple myeloma patients with normal (group 1) and impaired (group 2) renal function.

\begin{tabular}{lccc}
\hline & Group 1 & Group 2 & p-value \\
\hline Length right kidney $(\mathrm{cm})$ & $10.5 \pm 0.9$ & $10.1 \pm 1.6$ & n.s. \\
Length left kidney $(\mathrm{cm})$ & $10.9 \pm 1.1$ & $10.5 \pm 1.4$ & n.s. \\
Paren. width, right $(\mathrm{cm})$ & $1.7 \pm 0.2$ & $1.5 \pm 0.3$ & n.s. \\
Paren. width, left $(\mathrm{cm})$ & $1.6 \pm 0.2$ & $1.5 \pm 0.3$ & n.s. \\
Hyperechogenicity $(\mathrm{n} / \%)$ & $4(24 \%)$ & $0.71 \pm 0.08$ & 0.06 \\
RI right kidney & $0.67 \pm 0.06$ & $0.71 \pm 0.07$ & 0.15 \\
RI left kidney & $0.69 \pm 0.06$ & & \\
\hline
\end{tabular}

and the age of the patients [11-13]. In a own prospective study we determined values in a range between $0.59-0.63$ upon 78 healthy subjects and revealed that RI increases with age, about 0.001 per year [13], which is in accordance to others [14].

Renal vasoconstriction occurs under an activated renine-angiotensine-aldosterone system, for example in hepatic diseases like liver cirrhosis patients [15]. Recently, we revealed that renal RI measurement in liver cirrhosis correlates with the degree of vasoactive dysregulation and is an early sign of renal impairment as hints to a risk for hepatorenal syndrome. Liver cirrhosis patients with ascites (0.74) revealed significantly higher RI values than patients without ascites (0.67) and patients with elevated creatinine $(0.77)$ had higher values than those patients with normal creatinine (0.69) [16].

In multiple myeloma patients there was no significant difference between RI of those with elevated creatinine $(0.71)$ and those with normal creatinine (0.68). Obviously, the structural alterations of the kidneys do not lead to relevant vasoconstrictive effects.

In multiple myeloma patients kidneys are most frequently damaged directly by light chain components of the monoclonal immunoglobulin causing proximal tubular deletions and consecutive interstitial cast nephropathy [17]. Beside this, amyloidosis and light chain deposition disease are other causes of chronic renal impairment in multiple myeloma patients. As there is usually no therapeutic consequence invasive renal biopsy to determine the type of renal damage is not routinely performed and not recommended. Also in our patients, histology of renal disease was not obtained. Renal failure is a complication of high tumor burden and is a risk factor for a lower response to treatment and a poor prognosis [7, 18, 19]. Hypercalcemia, dehydration, infection, hyperuricemia and the use of nephrotoxic drugs also contributes to its aggravation [20] so that up to $3 \%$ of the patients require at least intermittend dialysis [3-8]. After effective chemotherapy approximately $25 \%$ of the patients recover normal kidney function [18]. The renal recovery correlates with the reduction of free light chains by plasma exchange [21].

This has also been shown for new therapeutic approaches in recurrent and refractory multiple myeloma. In these severe cases, Bortezomib for example, a selective and reversible proteasome inhibitor, provides clinical benefit of kidney function [22].
So far, no Doppler sonographical data exist whether vascular dysregulation occurs in myeloma kidneys and if these hypothesized effects would show reversibility under effective therapeutic regimen. Our results reveal only a non-significant elevation of renal RI in multiple myeloma patients compared to RI in healthy controls.

Analogous studies have already been performed for systemic lupus nephritis, another kind of interstitial nephropathy $[23,24]$. Herein, the data were controversial so that it remains unclear whether renal RI is elevated or not in these patients.

In contrast, significantly more myeloma patients with elevated creatinine and BUN as well as reduced GFR values reveal hyperechogenic kidney parenchyma than those with normal renal function, which is a morphological sign for interstitial nephropathy.

In summary, in spite of the limited number of patients measurement of renal RI does not give hints to a relevant vasoconstriction and is therefore no relevant parameter for the diagnosis of cast nephropathy of multiple myeloma patients. Routinely performed ultrasound examination should be more focussed on the qualification of parenchymal echogenicity.

Acknowledgement: This study was supported by grants of the Friedrich-Baur-Stiftung (Munich).

\section{REFERENCES}

1. Vivaldi P., Comotti C., Pedrazolli M. The kidney in multiple myeloma. The physiopathological and clinical aspects. Recenti Prog Med 1944;85(2):123-33.

2. Clark AD, Shetty A, Soutar R. Renal failure and multiple myeloma: pathogenesis and treatment of renal failure and management of underlying myeloma. Blood Rev 1999;13: $79-90$

3. Alexanian R., Barlogie B., Dixon D. Renal failure in multiple myeloma. Pathogenesis and prognostic implications. Arch Intern Med 1990;150:1693-1695

4. Johnson WJ., Kyle RA., Pineda AA., O’ Brien PC., Holley KE. Treatment of renal failure associated with multiple myeloma. Plamapheresis, hemodialysis, and chemotherapy. Arch Intern Med 1990;150:863-869

5. Torra R., Blade J., Cases A., Lopez-Pedret J., Montserrat E., Rozman C., Revert L. Patients with multiple myeloma requiring long-term dialysis: presenting features, response to therapy, and outcome in a series of 20 cases. Brit J Haematol 1995;91:854-859

6. Montseny JJ., Kleinknecht D., Meyrier A., Vanhille P., Simon P., Pruna A., Eladari D. Long-term outcome accord- 
ing to renal histological lesions in 118 patients with monoclonal gammopathies. Nephrol Dial Transplant 1998; 13:1438-1445

7. Knudsen LM, Hjorth M, Hippe E. Renal failure in multiple myeloma: reversibility and impact om the prognosis. Nordic myeloma study group.Eur J Haematol 2000; 65: $175-81$.

8. Badros A., Barlogie B., Siegel E., Roberts J., Langmaid C., Zangari M., Desikan R., Shaver MJ., Fassas A., McConnell S., Muwalla F., Barri Y., Anaissie E., Munshi N., Tricot G. Results of autologous stem cell transplant in multiple myeloma patients with renal failure. Brit J Haematol 2001;114:822-829

9. Dimopoulos MA., Kastritis E., Rosinol L., Bladé J., Ludwig $\mathrm{H}$. Pathogenesis and treatment of renal failure in multiple myeloma. Leukemia 2008;22(8):1485-93

10. Rivolta R, Maggi A, Cazzaniga M, Castagnone D, Panzeri A, Solenghi D, Lorenzano E, Quatro di Palo F, Salerno F: Reduction of renal cortical blood flow assessed by Doppler in cirrhotic patients with refractory ascites. Hepatology 1998; 28: 1235-1240.

11. Sacerdoti D, Gaiani S, Buonamico P, Merkel C, Zoli M, Bolondi L, Sabbà C. Interobserver and interequipment variability of hepatic, splenic and renal arterial Doppler Resistance Indices in normal subjects and patients with cirrhosis. Journal of Hepatology 1997; 27: 986-992

12. Lin ZY., Wang LY., Yu ML., Dai CY., Chen SC., Chuang WL., Hsieh MY., Tsai JF., Chang WY. Influence of age on intrarenal resistive index measurement in normal subjects. Abdom Imaging 2002;28:230-2

13. Kaiser C, Götzberger M, Landauer N, Dieterle C, Heldwein W, Schiemann U:Age dependency of intrarenal resistance index (RI) in healthy adults and patients with fatty liver disease. Eur J Med Res 2007; 12: 191-5.

14. Keogan MT., Kliewer MA., Hertzberg BS., DeLong DM., Tulper RH., Carroll BA. Renal resistive indixes: variability in Doppler US measurements in a healthy population. Radiology 1996;199:165-169

15. Platt J, Ellis JH, Rubin JM, Merion RM, Lucey MR. Renal duplex Doppler ulrasonography: A noninvasive predictor of kidney dysfunction and hepatorenal failure in liver disease. Hepatology 1994; 20: 362-369.

16. Götzberger M., Kaiser C., Landauer N., Dieterle C., Heldwein W., Schiemann U. Intrarenal resistance index for the assessment of early renal function impairment in patients with liver cirrhosis. Eur J Med Res 2008;13(8): 383-7

17. Bird JM., Fuge R., Sirohi B., Aperley JF., Hunter A., Snowden J., Mahendra P., Milligan D., Byrne J., Littlewood T., Fegan C., McQuaker G., Pagliuca A., Johnson P., Rahemtulla A., Morris C., Marks DI. The clinical outcome and toxicity of high-dose chemotherapy and autologous stem cell transplantation in patients with myeloma or amyloid and severe renal impairment: a British society of blood and marrow transplantation study. Brit J Haematol 2006;134:385-390
18. Bladé J., Fernadez-Llama P., Bosch F. et al. Renal failure in multiple myeloma : presenting features and predictos of outcome in 94 patients from a single institution. Arch Intern Med 1998;158:1889-1893

19. Sakhuja V., Jha V., Varma S. et al. Renal involvement in multiple myeloma: a 10-year study. Renal Fail 2000;22: 465-477

20. Smith A., Wisloff F., Samson D. Guidelines on the diagnosis and management of multiple myeloma. British J Haematol 2006;132:410-451

21. Leung N., Gertz MA., Zeldenrust SR., Rajkumar SV., Dispenzieri A., Fervenza FC., Kumar S., Lacy MQ., Lust JA., Greipp PR., Witzig TE., Hayman SR., Russell SJ., Kyle RA., Winters JL. Improvement of cast nephropathy with plasma exchange depends on the diagnosis and on reduction of serum free light chains. Kidney Int 2008;73(11):1282-8

22. Jagannath S., Barlogie B., Berenson JR., Singhal S., Alexanian R., Srkalovic G., Orlowski RZ., Richardson PG., Anderson J., Nix D., Esseltine DL., Anderson KC. Bortezomib in recurrent and /or refractory multiple myeloma. Cancer 2005;103:1195-200

23. Ozbek SS., Büyükberber S., Tolunay O., Erden MI., Aytac SK., Olmez U. Image directed color Doppler ultrasonography of kidney in systemic lupus nephritis. J Clin Ultrasound 1995;23(1):17-20

24. Platt J, Rubin J, Ellis J. Lupus nephritis: Predictive value of conventional and Doppler ultrasound and comparison with serologic and biopsy parameters. Radiology 1997; 203: 82-86

25. Aikimbaev KS, Canataroglu A, Ozbek S, Usal A. Renal vascular resistance in progressive systemic sclerosis: Evaluation with duplex Doppler ultrasound. Angiology 2001; 52: 697-701

Received: December 15, 2009 / Accepted: February 18, 2010

Address for correspondence:

PD Dr. med. U. Schiemann

Klinik für Allgemeine Innere Medizin/ Notfallzentrum

Inselspital Bern (Universitätsspital)

Freiburgstrasse

3010 Bern

Switzerland

Tel.: $\quad++41-31-6322111$

Fax: $\quad++41-31-6324814$

E-mail: uwe.schiemann@insel.ch 